\title{
Propriedades funcionais tecnológicas das fibras de soja, aveia e trigo e produtos de soja com adição de fibras e fermentados com cultura de kefir
}

\section{Technological functional properties of soy, oat and wheat fibers and soy products with added fibers and fermented with kefir culture}

\author{
Tahis Regina Baú ${ }^{1}$; Letícia Cardoso da Silva ${ }^{2}$; Sandra Garcia ${ }^{3}$; Elza Iouko Ida ${ }^{3 *}$
}

\section{Resumo}

O objetivo deste trabalho foi caracterizar as propriedades funcionais tecnológicas das fibras de soja, aveia e trigo e avaliar os produtos de soja com adição de fibras e fermentados com cultura de kefir. As fibras foram caracterizadas quanto a composição química, $\mathrm{pH}$, capacidade de adsorção e absorção de água, capacidade de absorção de óleo e volume de intumescimento. Foram desenvolvidos produtos de soja com adição de 3\% de fibra de soja, aveia e trigo e fermentados com cultura de kefir. Os produtos fermentados foram caracterizados quanto o $\mathrm{pH}$, acidez, sinerese, viscosidade e firmeza, contagem de Lactococcus lactis e análise das condições higiênico-sanitárias. A fibra de soja apresentou maior teor de proteínas e lipídios, $\mathrm{pH}$, capacidade de adsorção e adsorção de água. A fibra de trigo apresentou maior capacidade de absorção de óleo. A adição de fibras de soja, aveia e trigo influenciaram o pH, acidez, sinerese, viscosidade e firmeza dos produtos de soja fermentados com kefir. A menor sinerese e maior viscosidade e firmeza foram obtidos para os produtos fermentados com fibra de soja. O crescimento de Lactococcus lactis foi favorecido no produto fermentado com adição de fibra de soja. Por conterem suficiente contagem, ou seja, acima de 6 log UFC.g ${ }^{-1}$ de Lactococcus lactis, os produtos fermentados podem ser considerados probióticos. Os produtos apresentaram condições higiênico-sanitárias satisfatórias.

Palavras-chave: Produto probiótico, viscosidade, firmeza, sinerese

\begin{abstract}
The objective of this study was to characterize the technological functional properties of soy, oats and wheat fibers and evaluate soy products with added fiber and fermented with kefir. The fibers were characterized with regard to $\mathrm{pH}$, water adsorption capacity, water and oil absorption capacity and swelling volume. Soy products were developed with the addition of 3\% soy fiber, oat and wheat and fermented with kefir. The fermented products were characterized for $\mathrm{pH}$, acidity, syneresis, viscosity and firmness, Lactococcus lactis counts and analysis of sanitary conditions. The soy fiber showed a higher content of protein and lipids, $\mathrm{pH}$, water adsorption and adsorption capacity. The wheat fiber showed a higher oil absorption capacity. The addition of soy, oat and wheat fibers influence $\mathrm{pH}$, acidity, syneresis, viscosity and firmness of fermented soy products with kefir. The lowest syneresis and highest viscosity and firmness were obtained with the soy fermented products with soy fiber. The addition of soy fiber stimulated Lactococcus lactis growth. The fermented soy products had enough Lactococcus lactis counts to be considered probiotics. The products presented satisfactory hygienic sanitary conditions.
\end{abstract}

Key words: Probiotic product, fiber, viscosity, firmness, syneresis

\footnotetext{
1 Doutoranda em Ciência de Alimentos, Dept ${ }^{\circ}$ de Ciência e Tecnologia de Alimentos, Universidade Estadual de Londrina, UEL, Londrina, PR. E-mail: tahisbau@yahoo.com.br

2 Discente do curso de Farmácia, UEL, Londrina, PR. Bolsista Iniciação Científica. E-mail: letícia_card0s0@hotmail.com

3 Prof ${ }^{\mathrm{a}} \mathrm{s}$ Dr ${ }^{\mathrm{a}} \mathrm{s}$. do Dept $^{\mathrm{o}}$ de Tecnologia de Alimentos, UEL, Londrina, PR. E-mail: sgarcia@uel.br; elida@uel.br

*Autor para correspondência
} 


\section{Introdução}

O mercado de alimentos funcionais e novos ingredientes aumentaram significativamente devido a vários fatores relacionados com a saúde, estilo de vida, nível de informação dos consumidores, mercado altamente competitivo, etc (GRANATO et al., 2010; SIRÓ et al., 2008). O desenvolvimento de alimentos funcionais a base de vegetais é desafiador e visa cumprir a expectativa do consumidor por produtos com boas características sensoriais (SHAH, 2007) e que apresentem componentes importantes para a saúde, como as fibras. Além disso, fatores como a intolerância à lactose, alergias às proteínas do leite e a presença de colesterol, tornou essencial o desenvolvimento de novos produtos para atender este segmento (GRANATO et al., 2010).

A fermentação de produtos vegetais tem como intuito atender as atuais demandas dos consumidores por produtos não-lácteos funcionais. Dentre os microrganismos utilizados na produção de alimentos fermentados, destaca-se a cultura de kefir, que é composta por uma complexa microbiota simbiótica de bactérias ácido lácticas, acéticas e leveduras que são responsáveis pela fermentação ácida-alcoólica (IRIGOYEN et al., 2005; GUZEL-SEYDIM et al., 2011). Em virtude da complexidade microbiana e dos efeitos benéficos derivados do seu consumo, o kefir pode ser considerado uma fonte adequada de microorganismos com potencial probiótico (ROMANIN et al., 2010), sendo apontado como um alimento probiótico e funcional (LOPITZ-OTSOA et al., 2006; SIMOVA et al., 2002), além de apresentar um papel potencialmente benéfico na redução do risco de doenças crônicas degenerativas. O kefir é tradicionalmente um produto elaborado a partir do leite (GUZEL-SEYDIM et al., 2011), sendo que sua utilização na fermentação de substratos vegetais, como o extrato de soja, representa uma alternativa inovadora no desenvolvimento de novos produtos.

A adição de fibras em variados tipos de produtos também é crescente no desenvolvimento de alimentos funcionais. A adição de fibras naturais em produtos fermentados apresenta benefícios, pois pode contribuir para uma melhor textura e firmeza e reduzir a sinerese, o que resulta em um produto com propriedades sensoriais aceitáveis (McCANN; FABRE; DAY, 2011). Durante a fermentação algumas características do extrato de soja podem ser influenciadas pela adição de fibras, como: a produção de ácido, a textura, o desenvolvimento microbiológico e a estabilidade no armazenamento (APORTELA-PALACOIS; SOSA-MORALES; VELEZ-RUIZ, 2005; VASILJEVIC; KEALY; MISHRA, 2007; SENDRA et al., 2010; ZARE et al., 2011). Em produtos fermentados como o iogurte, foram descritos a adição de fibra de maçã, trigo, bambu e inulina com alteração nas características reológicas e sensoriais (DELLO STAFFOLO et al., 2004). Em produtos fermentados de soja não foram encontrados estudos envolvendo a adição de fibras.

O objetivo deste trabalho foi caracterizar as propriedades funcionais tecnológicas das fibras de soja, aveia e trigo e avaliar os produtos de soja com adição de fibras e fermentados com cultura de kefir.

\section{Materiais e Métodos}

\section{Matéria-prima e cultura starter}

O extrato de soja foi preparado com a cultivar de soja BRS 257, livre de lipoxigenases, doada pela empresa Sementes Paraná, Mauá da Serra, PR. Para formulação do produto fermentado de soja, foram utilizados os seguintes ingredientes comerciais: fibra de soja, fibra de aveia, fibra de trigo, sacarose, antiespumante, aromatizante artificial de leite e aromatizante artificial de baunilha.

Para fermentação foi utilizada cultura starter de kefir liofilizada (Sacco ${ }^{\circledR}$-Lyofast MT 036 LV) contendo $5 \mathrm{UC} / \mathrm{envelope,} \mathrm{composta} \mathrm{por} \mathrm{linhagens}$ mistas de Lactococcus lactis ssp. lactis, Lactococcus lactis spp. lactis biovar diacetylactis, Lactobacillus brevis, Leuconostoc e Saccharomyces cerevisiae. 
Preparo do extrato de soja e produto fermentado de soja com adição de fibras

O extrato de soja foi preparado após seleção e lavagem dos grãos de soja. Os grãos na proporção 1:10 (peso:volume; grãos de soja:água) foram macerados por $14 \mathrm{~h}$, triturados (Liquidificador Britânia, Mod. AS25F, Brasil) e filtrados para obter o extrato de soja e o resíduo foi descartado.

Os produtos fermentados foram desenvolvidos conforme a Figura 1. As formulações contendo $87,7 \%$ de extrato de soja, $3,0 \%$ de fibras, $9,0 \%$ de sacarose e $0,1 \%$ de antiespumante foram submetidas ao tratamento térmico a $95^{\circ} \mathrm{C}$ por 15 min segundo recomendações de Ferragut et al. (2009) e após o resfriamento até $25^{\circ} \mathrm{C}$, foi adicionado $0,2 \%$ de aromatizantes de leite e baunilha e envasadas em frascos de vidro de $600 \mathrm{~mL}$. Em seguida, foram fermentadas a $25^{\circ} \mathrm{C}$ com cultura de kefir $(0,01$ $\mathrm{UC} / \mathrm{L}$ ) até atingir o $\mathrm{pH}$ igual a 4,5 $\pm 0,1$. Após a fermentação, os frascos foram resfriados até $4^{\circ} \mathrm{C}$, homogeneizados por 6 min em velocidade constante (Homogeneizador Contrac, Mod. 1000, Brasil) e armazenados no mínimo por $12 \mathrm{~h}$ para realização das análises.

Figura 1. Desenvolvimento dos produtos de soja com adição de fibras e fermentados com kefir.

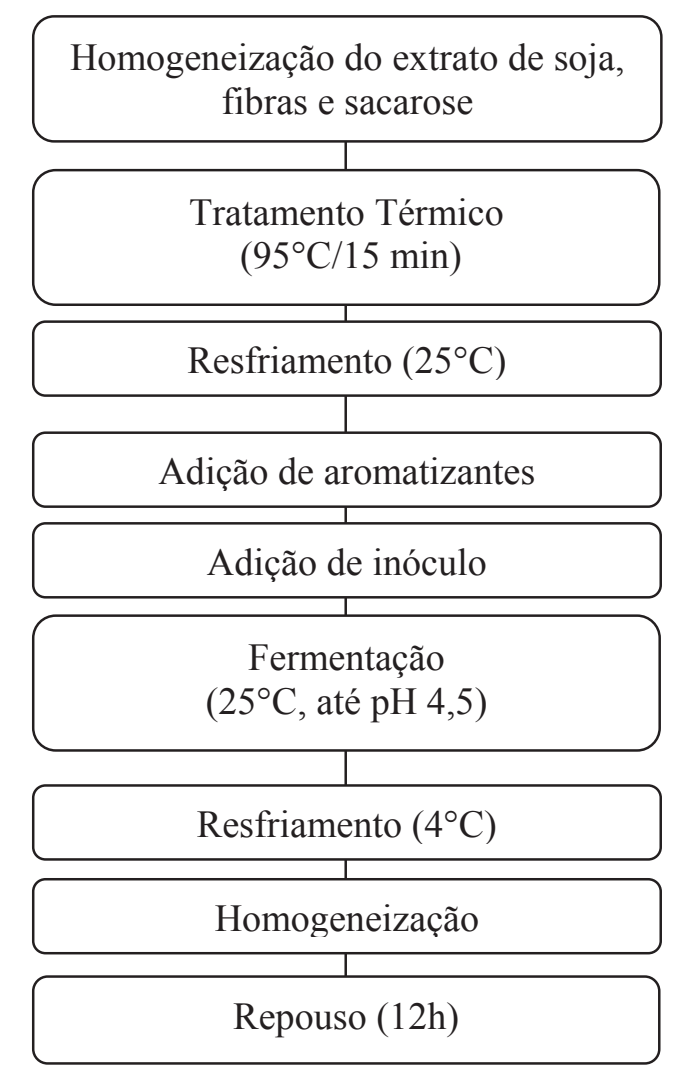

Fonte: Elaboração dos autores.

Foram realizadas quatro formulações com adição de fibras conforme segue: Formulação 1 contendo $3 \%$ de fibra de soja; Formulação 2 contendo $3 \%$ de fibra de aveia; Formulação 3 contendo $3 \%$ de fibra de trigo. A formulação C não foi adicionada de fibras e o volume de extrato de soja nesta formulação foi ajustado para $90,7 \%$. 
Composição química e propriedades funcionais tecnológicas das fibras de soja, aveia e trigo e produtos fermentados com adição destas fibras

A composição química das fibras de soja, aveia e trigo foi determinada segundo a AOAC (2006) e expressa em base seca (b.s.). $\mathrm{O}$ pH das fibras e dos produtos fermentados com adição de fibras foi determinado em potenciômetro digital (Hanna, Mod. HI 223, Estados Unidos) e as propriedades funcionais tecnológicas das fibras foram medidas pela capacidade de adsorção de água (CDA) conforme Vázquez-Ovando et al. (2009), capacidade de absorção de água (CAA) e capacidade de absorção de óleo (CAO) conforme Okezie e Bello (1988) e volume de intumescimento (VI) conforme Robertson et al. (2000).

A acidez dos produtos fermentados com adição de fibras foi medida por titulação com $\mathrm{NaOH}$ $0,1 \mathrm{M}$ e expressa em $\mathrm{g}$ de ácido láctico. $100 \mathrm{~g}^{-1} \mathrm{de}$ produto. A viscosidade dos produtos fermentados foi determinada utilizando o viscosímetro digital (Brookfield, Mod. DV II+, Estados Unidos) com spindle 4, velocidade de $12 \mathrm{rpm}$, em $600 \mathrm{~mL}$ de amostra a $4 \pm 1^{\circ} \mathrm{C}$ e o resultado foi expresso em centipoise. A sinerese, em cinco replicatas, foi medida nos produtos fermentados conforme modificação da metodologia descrita por Guirguis, Broome e Hickey (1984), no qual foi utilizado o tecido tunil sobreposto sob uma peneira para drenagem. A sinerese foi expressa como $\mathrm{mL}$ de exsudato. $100 \mathrm{~g}^{-1}$ de amostra. A firmeza dos produtos fermentados foi expressa em Newton $(\mathrm{N})$ e avaliada pelas medidas realizadas no texturômetro (Stable Micro Systems, Mod. TA-XT2i, Reino Unido), com probe cilíndrico de acrílico $\mathrm{P} 25 / \mathrm{L}$, profundidade de compressão de $10 \mathrm{~mm}$, velocidade de compressão do sensor de $2 \mathrm{~mm} . \mathrm{s}^{-1}$, força de trigger de $0,05 \mathrm{~N}$ e tempo de $0,5 \mathrm{~s}$.
Caracterização microbiológica dos produtos fermentados

Para caracterização microbiológica dos produtos fermentados com adição de fibras foi realizada a contagem de Lactococcus lactis em ágar M17 (pH $7,2 \pm 0,2)$, suplementado com cicloheximida (200 mg. $L^{-1}$ ), por meio da semeadura em superfície e incubação das placas a $30^{\circ} \mathrm{C}$ por $48 \mathrm{~h}$, em anaerobiose (IRIGOYEN et al., 2005). O resultado foi expresso em $\log$ UFC.g-1 do produto. A confirmação foi realizada por coloração de Gram. Foi realizada a contagem deste microrganismo com o intuito de verificar se o produto poderia ser considerado como probiótico, segundo a legislação vigente.

Para avaliação das condições higiênicosanitárias foram realizadas contagem de Bacillus cereus, contagem de Coliformes a $45^{\circ} \mathrm{C}$ e pesquisa de Salmonella sp, seguindo a metodologia descrita na Instrução Normativa $n^{\circ} 62$ de 26 de agosto de 2003 (BRASIL, 2003).

\section{Análise dos dados}

Os dados foram submetidos a Análise de Variância-ANOVA one way e Teste de Tukey $(\mathrm{p}<0,05)$ para comparação de médias, utilizando o software Statistica 7.0 (STATSOFT, 2004).

\section{Resultados e Discussão}

As fibras de soja, aveia e trigo apresentaram diferenças na composição química (Tabela 1) e nas propriedades funcionais tecnológicas (Tabela 2), devido as suas respectivas origens e diferentes formas de processamento para sua obtenção. O teor de cinzas nas fibras apresentou diferenças (Tabela 1). O teor de proteínas e lipídios na fibra de soja foi maior do que nas fibras de aveia e trigo. O teor de fibras totais, solúveis e insolúveis foi maior na fibra de trigo, seguida da fibra de aveia e soja. 
Tabela 1. Composição química em base seca das fibras de soja, aveia e trigo.

\begin{tabular}{lccc}
\hline Composição química $\left(\mathbf{g . 1 0 0} \mathbf{~ g}^{-1}\right)$ & Fibra de soja & Fibra de Aveia & Fibra de trigo \\
Cinzas & $4,36 \pm 0,01^{\mathrm{b}}$ & $3,96 \pm 0,01^{\mathrm{c}}$ & $5,76 \pm 0,01^{\mathrm{a}}$ \\
Proteínas & $41,5 \pm 0,37^{\mathrm{a}}$ & $1,98 \pm 0,23^{\mathrm{b}}$ & $0,00 \pm 0,00^{\mathrm{c}}$ \\
Lipídios & $1,36 \pm 0,21^{\mathrm{a}}$ & $0,40 \pm 0,08^{\mathrm{b}}$ & $0,40 \pm 0,08^{\mathrm{b}}$ \\
Fibras solúveis & $1,44 \pm 0,30^{\mathrm{b}}$ & $1,10 \pm 0,13^{\mathrm{b}}$ & $2,77 \pm 0,20^{\mathrm{a}}$ \\
Fibras insolúveis & $43,78 \pm 0,61^{\mathrm{c}}$ & $83,55 \pm 0,15^{\mathrm{b}}$ & $90,05 \pm 0,15^{\mathrm{a}}$ \\
Fibras totais & $45,22 \pm 0,65^{\mathrm{c}}$ & $84,65 \pm 0,18^{\mathrm{b}}$ & $92,82 \pm 0,30^{\mathrm{a}}$ \\
\hline
\end{tabular}

Médias \pm desvio padrão nas mesmas linhas acompanhadas de letras iguais não diferem estatisticamente $(\mathrm{p} \leq 0,05)$ pelo teste de Tukey.

Fonte: Elaboração dos autores.

Tabela 2. Propriedades funcionais tecnológicas das fibras de soja, aveia e trigo.

\begin{tabular}{lccc}
\hline & Fibra de Soja & Fibra de Aveia & Fibra de Trigo \\
\hline pH & $6,86 \pm 0,03^{\mathrm{a}}$ & $5,19 \pm 0,01^{\mathrm{c}}$ & $5,88 \pm 0,04^{\mathrm{b}}$ \\
CDA (g de água/g amostra b.s.) & $0,284 \pm 0,006^{\mathrm{a}}$ & $0,153 \pm 0,005^{\mathrm{b}}$ & $0,146 \pm 0,003^{\mathrm{b}}$ \\
CAA (g de água/g amostra b.s.) & $5,44 \pm 0,18^{\mathrm{a}}$ & $2,96 \pm 0,28^{\mathrm{c}}$ & $4,52 \pm 0,38^{\mathrm{b}}$ \\
CAO (g de óleo/g amostra b.s.) & $1,66 \pm 0,22^{\mathrm{b}}$ & $1,96 \pm 0,20^{\mathrm{b}}$ & $3,20 \pm 0,20^{\mathrm{a}}$ \\
VI (mL/g amostra b.s.) & $9,7 \pm 0,48^{\mathrm{a}}$ & $3,2 \pm 0,31^{\mathrm{c}}$ & $7,0 \pm 0,07^{\mathrm{b}}$ \\
\hline
\end{tabular}

Médias \pm desvio padrão nas mesmas linhas acompanhadas de letras iguais não diferem estatisticamente $(\mathrm{p} \leq 0,05)$ pelo teste de Tukey. CDA: capacidade de adsorção de água; CAA: capacidade de absorção de água; CAO: capacidade de absorção de óleo; VI: volume de intumescimento.

Fonte: Elaboração dos autores.

$\mathrm{O} \mathrm{pH}$ foi maior na fibra de soja, seguida da fibra de trigo e aveia (Tabela 2). As CDA, CAA e VI da fibra de soja foram maiores e diferiram das fibras de aveia e trigo, pois o teor de proteínas da fibra de soja foi maior do que o teor de proteínas na fibra de aveia, e a fibra de trigo não apresentarou proteínas (Tabelas 1 e 2). A capacidade de hidratação das proteínas está relacionada, em parte, à quantidade de resíduos de aminoácidos carregados, sendo também dependente da conformação da sua molécula. A fibra de trigo apresentou maior CAA e VI do que a fibra de aveia (Tabela 2) uma vez que a propriedade de hidratação das fibras também pode ser influenciada pela quantidade das fibras solúveis. A propriedade de hidratação das fibras também pode estar relacionada com a estrutura, composição química dos polissacarídeos, porosidade, tamanho de partícula, força iônica, $\mathrm{pH}$, temperatura, etc (ELLEUCH et al., 2011). Além das propriedades de hidratação, as fibras possuem a capacidade de reter o óleo. A fibra de trigo apresentou maior CAO do que a fibra de soja ou aveia (Tabela 2). Assim, as elevadas CAA e CAO da fibra de trigo (Tabela 2), possibilitam a sua utilização como um ingrediente em produtos fermentados. Os valores de CAO de 11,3 g de óleo/g de fibras foram descritos para bagaço de cana-deaçúcar (SANGNARK; NOOMHORM, 2003), de 5,5 para fibras de cenoura (EIM et al., 2008) e 1,09 para fibra concentrada de pêssego (ELLEUCH et al., 2008). Os valores de CAA de 7,5 g de água/g de fibras de bagaço de cana-de-açúcar, 18,6 para fibras de cenoura e 12,1 para fibra de pêssego foram relatadas respectivamente pelos mesmos autores. As fibras com elevada CAA podem ser aplicadas em alguns produtos alimentícios para evitar a sinerese e modificar a viscosidade e textura. Enquanto que as fibras com elevada CAO podem ser utilizadas em alimentos ricos em gordura e emulsões, pois facilitam a estabilização (GRIGELMO-MIGUEL, CARRERAS-BOLADERAS; MARTINABELLOSO, 1999). 
Os produtos fermentados com adição de fibra de soja, aveia e trigo apresentaram diferenças nas propriedades funcionais tecnológicas (Tabela 3). Os produtos fermentados de soja com kefir apresentaram $\mathrm{pH}$ que variou de 4,46 a 4,62, cuja faixa de $\mathrm{pH}$, segundo Chumchuere e Robinson (1999), foi considerada como ótima para formação do gel de extrato de soja. $\mathrm{O}$ pH do produto fermentado de soja contendo fibra de aveia foi significativamente maior e indica que não está relacionado com o $\mathrm{pH}$ do ingrediente fibra de aveia (Tabela 2), provavelmente devido à pequena quantidade de $3 \%$ que foi adicionada na formulação do produto fermentado. A acidez da formulação contendo fibra de aveia não diferiu da formulação sem adição de fibras, indicando pouca ou nenhuma influência sobre a produção de ácido do produto fermentado (Tabela 3). A adição de fibra de soja promoveu o aumento na produção de ácido láctico, seguido da fibra de trigo. A capacidade de algumas fibras em aumentar a acidez de produtos fermentados também foi descrita quando se utilizou a fibra de laranja (GARCÍA- PÉREZ et al., 2006; LARIO et al., 2004) soja, arroz, milho, aveia e beterraba (FERNANDEZ-GARCIA; McGREGOR, 1997) e isto pode ser devido o fornecimento nutrientes ou fatores estimulantes para cultura starter. Valores similares de $\mathrm{pH}$ foram obtidos por Camargo et al. (2000) que desenvolveram uma bebida a base de soro de leite e isolado protéico de soja, que também obtiveram valores mais elevados de acidez.

Tabela 3. Propriedades funcionais tecnológicas dos produtos de soja com adição de fibras e fermentados com kefir.

\begin{tabular}{cccccc}
\hline Formulaçã $^{*}$ & $\mathbf{p H}$ & $\begin{array}{c}\text { Acidez } \\
\left(\mathrm{g} \text { ac láctico.100 } \mathrm{g}^{-1}\right)\end{array}$ & $\begin{array}{c}\text { Sinerese } \\
\left(\mathrm{mL} \cdot 100 \mathrm{~g}^{-1}\right)\end{array}$ & $\begin{array}{c}\text { Viscosidade } \\
(\text { centipoise })\end{array}$ & $\begin{array}{c}\text { Firmeza } \\
(\mathrm{N})\end{array}$ \\
\hline $\mathbf{1}$ & $4,49 \pm 0,01^{\mathrm{b}}$ & $0,514 \pm 0,01^{\mathrm{a}}$ & $8,1 \pm 0,5^{\mathrm{d}}$ & $392,08 \pm 9,12^{\mathrm{a}}$ & $0,204 \pm 0,01^{\mathrm{a}}$ \\
$\mathbf{2}$ & $4,62 \pm 0,00^{\mathrm{a}}$ & $0,370 \pm 0,01^{\mathrm{c}}$ & $21,2 \pm 1,3^{\mathrm{b}}$ & $160,00 \pm 8,97^{\mathrm{c}}$ & $0,157 \pm 0,01^{\mathrm{b}, \mathrm{c}}$ \\
$\mathbf{3}$ & $4,53 \pm 0,01^{\mathrm{b}}$ & $0,412 \pm 0,01^{\mathrm{b}}$ & $15,7 \pm 0,6^{\mathrm{c}}$ & $267,83 \pm 23,04^{\mathrm{b}}$ & $0,170 \pm 0,01^{\mathrm{b}}$ \\
$\mathbf{C}$ & $4,46 \pm 0,03^{\mathrm{b}}$ & $0,374 \pm 0,01^{\mathrm{c}}$ & $24,5 \pm 2,8^{\mathrm{a}}$ & $167,48 \pm 11,93^{\mathrm{c}}$ & $0,152 \pm 0,01^{\mathrm{c}}$ \\
\hline
\end{tabular}

Médias \pm desvio padrão na mesma coluna acompanhada de letras iguais não diferem estatisticamente $(\mathrm{p} \leq 0,05)$. *Formulações: 1 (produto adicionado de fibra de soja); 2 (produto adicionado de fibra de aveia); 3 (produto adicionado de fibra de trigo); $\mathrm{C}$ (produto sem adição de fibra).

Fonte: Elaboração dos autores.

A sinerese, segundo Zare et al. (2011), foi definida como a separação espontânea do soro do produto fermentado. A adição de fibras também influencia a sinerese dos produtos fermentados, sendo que a formulação contendo fibra de soja apresentou menor sinerese, seguida da formulação contendo fibra de trigo e aveia (Tabela 3 ). A sinerese dos produtos de soja fermentados com kefir, em geral, foi baixa e similar aos índices obtidos por Kovalenko e Briggs (2002). Os resultados também foram similares aos obtidos por Magenis et al. (2006) que observaram que quanto menor o teor protéico da formulação maior o valor de sinerese. O produto fermentado sem adição de fibras (formulação controle) apresentou maior valor de sinerese, conforme confirmado também por Ünal, Metin e I-Ikli (2003). A formulação controle, por não ser adicionada de fibras, apresentou aproximadamente $3 \%$ de sólidos a menos que as formulações adicionadas de fibras. As principais causas da separação do soro são as rápidas acidificações, elevadas temperatura de incubação, tratamento térmico excessivo, baixo teor de sólidos totais, agitação durante ou logo após a formação do coágulo e baixa produção de ácido com pH de 4,8 (LUCEY, 2001). 
A viscosidade e a firmeza foram maiores no produto fermentado com adição de fibra de soja, sendo que os menores valores foram obtidos para a formulação contendo fibra de aveia e para a formulação controle (Tabela 3). Estas características podem estar relacionadas com a maior CDA, CAA e VI da fibra de soja (Tabela 2). Ainda, a fibra de soja apresentou maior conteúdo de proteínas (Tabela 1) e, segundo Damodaran, Parkin e Fennema (2010), os polímeros solúveis de alta massa molar, como as proteínas, aumentam a viscosidade, mesmo em concentrações baixas e a viscosidade segue uma relação exponencial com a concentração de proteína. Segundo Magenis et al. (2006), em geral, a viscosidade de produtos fermentados é uma característica muito importante na qualidade do produto. A adição de diferentes fontes de fibras como de bambu, maçã e trigo ou inulina em iogurte afetaram a viscosidade e a firmeza de iogurte (DELLO STAFFOLO et al., 2004). Sendra et al. (2010) observaram que a adição de $1 \mathrm{~g} .100 \mathrm{~mL}^{-1}$ de fibra de laranja em iogurte promoveu aumento na firmeza do gel e cremosidade do produto. Por outro lado, a adição de fibras naturais em produtos fermentados contribui com a firmeza e diminuição da sinerese (McCANN; FABRE; DAY, 2011).
Os probióticos são micro-organismos vivos que, quando administrados em quantidades adequadas, afetam positivamente a saúde do hospedeiro (FAO/WHO, 2001). Altas contagens desses micro-organismos em produtos são sugeridas para compensar a possível redução durante a passagem pelo estômago e intestino. A adição de fibras nos produtos fermentados desoja comkefirinfluenciouno crescimento de Lactococcus lactis (Tabela 4), sendo que a formulação contendo fibra de soja apresentou maior contagem do probiótico. Conforme previsto na legislação brasileira todas as formulações (Tabela 4) são probióticas devido a contagem mínima de 6 log UFC.g ${ }^{-1}$ (BRASIL, 2007). O extrato de soja é um substrato adequado para o crescimento de Lactococcus lactis (BEASLEY; TUORILA; SARIS, 2003; CHAMPAGNE et al., 2009). A formulação contendo fibra de soja e, conseqüentemente, maior quantidade de proteínas pode ter estimulado o crescimento destes microrganismos devido a maior capacidade tamponante, conforme observado também por Champagne et al. (2009). Em produtos fermentados, os efeitos da adição de vários tipos de ingredientes sobre a atividade de culturas láticas dependem do tipo e da concentração destes ingredientes (CHAMPAGNE et al., 2009) e de outros fatores como a presença de prebióticos ou micronutrientes.

Tabela 4. Contagem de Lactococcus lactis nos produtos de soja com adição de fibras e fermentados com kefir.

\begin{tabular}{cc}
\hline Formulação* & Contagem de Lactococcus lactis \\
$(\log$ UFC.g-1)
\end{tabular}

Médias \pm desvio padrão na mesma coluna acompanhada de letras iguais não diferem estatisticamente $(p \leq 0,05)$. ${ }^{*}$ Formulações: 1 (produto adicionado de fibra de soja); 2 (produto adicionado de fibra de aveia); 3 (produto adicionado de fibra de trigo); $\mathrm{C}$ (produto sem adição de fibra).

Fonte: Elaboração dos autores. 
Todas as formulações apresentaram condições higiênico-sanitárias satisfatórias (Tabela 5). As formulações de produtos fermentados não apresentaram contagens de Bacillus cereus, Coliformes a $45^{\circ} \mathrm{C}$ ou presença de Salmonella sp e estão de acordo com os limites estabelecidos pela legislação vigente (BRASIL, 2001).
A caracterização funcional tecnológica das fibras é uma etapa importante para sua posterior incorporação em alimentos, uma vez que é determinante nas características físicas, químicas e microbiológicas dos produtos obtidos. Além disso, a influência que os ingredientes exercem sobre as características do produto final representa um fator de investigação que deve ser considerado no desenvolvimento de novos produtos fermentados.

Tabela 5. Características higiênico-sanitárias dos produtos de soja com adição de fibras e fermentados com kefir.

\begin{tabular}{cccc}
\hline Formulações* & $\begin{array}{c}\text { Cont. Bacillus cereus } \\
\left(\text { UFC. } \mathrm{g}^{-1}\right)\end{array}$ & $\begin{array}{c}\text { Cont. Colifomes } 45^{\circ} \mathbf{C} \\
\left(\text { UFC. } \mathrm{g}^{-1}\right)\end{array}$ & Pesq. Salmonella sp \\
\hline $\mathbf{1}$ & $<10^{2 \mathrm{a}}$ & $<10^{\mathrm{a}}$ & Ausência em $25 \mathrm{~g}^{\mathrm{a}}$ \\
$\mathbf{2}$ & $<10^{2 \mathrm{a}}$ & $<10^{1 \mathrm{a}}$ & Ausência em $25 \mathrm{~g}^{\mathrm{a}}$ \\
$\mathbf{3}$ & $<10^{2 \mathrm{a}}$ & $<10^{1 \mathrm{a}}$ & Ausência em $25 \mathrm{~g}^{\mathrm{a}}$ \\
$\mathbf{C}$ & $<10^{2 \mathrm{a}}$ & $<10^{1 \mathrm{a}}$ & Ausência em $25 \mathrm{~g}^{\mathrm{a}}$ \\
\hline
\end{tabular}

Médias \pm desvio padrão na mesma coluna acompanhada de letras iguais não diferem estatisticamente $(\mathrm{p} \leq 0,05)$. *Formulações: 1 (produto adicionado de fibra de soja); 2 (produto adicionado de fibra de aveia); 3 (produto adicionado de fibra de trigo); $\mathrm{C}$ (produto sem adição de fibra).

Fonte: Elaboração dos autores.

A adição de fibras de soja, aveia e trigo influenciaram o $\mathrm{pH}$, acidez, sinerese, viscosidade e firmeza dos produtos de soja fermentados com kefir. A menor sinerese e maior viscosidade e firmeza foram obtidos para os produtos fermentados com fibra de soja. O crescimento de Lactococcus lactis foi favorecido no produto fermentado com adição de fibra de soja. Pela contagem de Lactococcus lactis os produtos fermentados podem ser considerado probióticos. Os produtos apresentaram condições higiênico-sanitárias satisfatórias. Para estudos futuros, será necessário realizar a análise de aceitação sensorial e o perfil de compostos voláteis dos produtos.

\section{Agradecimentos}

Os autores agradecem a Fundação Araucária/ CNPq-Pronex, T.R.B pela concessão de bolsas de estudos e E.I.I pela Bolsa Produtividade PQ/CNPq.

\section{Referências}

ASSOCIATION OF OFFICIAL ANALYTICAL CHEMISTS - AOAC. Official methods of analysis. 18. ed. Washington, DC: Association of Official Analytical Chemistry, v. 1, v. 2, 2006.

APORTELA-PALACOIS, A.; SOSA-MORALES, M. E.; VELEZ-RUIZ, J. F. Rheological and physicochemical behavior of fortified yogurt, with fiber and calcium. Journal of Texture Studies, v. 36, n. 3, p. 333-349, 2005.

BEASLEY, S.; TUORILA, H.; SARIS, P. E. J. Fermented soymilk with a monoculture of Lactococcus lactis. International Journal of Food Microbiology, v. 81, n. 2, p. 159-162, 2003.

BRASIL, Ministério da Saúde. Agência Nacional de Vigilância Sanitária. Alimentos com alegações de propriedades funcionais e ou de saúde, novos alimentos/ ingredientes, substâncias bioativas e probióticos. Atualizado em agosto de 2007. IX - Lista das alegações de propriedades funcionais aprovadas. Disponível em: $<$ http://anvisa.gov.br/alimentos/comissões/ tecno_lista_ alega.htm>. Acesso em: 18 abr. 2007.

Métodos Analíticos Oficiais para Análises Microbiológicas para Controle de Produtos de Origem 
Animal e Água (Anexo I), Instrução Normativa $\mathrm{N}^{\circ} 62$, de 26 de agosto de 2003. Ministério da Agricultura, Pecuária e Abastecimento. Diário Oficial [da] União, Brasília, 18 de Setembro de 2003.

Regulamento Técnico Sobre Padrões Microbiológicos Para Alimentos, Resolução - RDC n 12, de 2 de janeiro de 2001. Ministério da Agricultura, Pecuária e Abastecimento. Diário Oficial [da] União, Brasília, 2 de Janeiro de 2001.

CAMARGO, D. S.; ALVES, G.; GARCIA, S.; MIZUBITI, I. Bebida fermentada à base de soro de leite e isolado protéico de soja. Semina: Ciências Agrárias, Londrina, v. 21, n. 1, p. 45-51, 2000.

CHAMPAGNE, C. P.; GREEN-JOHNSON, J.; RAYMOND, Y.; BARRETTE, J.; BUCKLEY, N. Selection of probiotic bacteria for the fermentation of a soy beverage in combination with Streptococcus thermophilus. Food Research International, v. 42, n. 5-6, p. 612-621, 2009.

CHUMCHUERE, S.; ROBINSON, R. K. Selection of starter cultures for the fermentation of soya milk. Food Microbiology, London, v. 16, n. 2, p. 129-137, 1999.

DAMODARAN, S.; PARKIN, K. L.; FENNEMA, O. R. Química de alimentos de Fennema. 4. ed. Porto Alegre: Artmed, 2010.

DELLO STAFFOLO, M.; BERTOLA, N.; MARTINO, M.; BEVILACQUA, A. Influence of dietary fiber addition on sensory and rheological properties of yogurt. International Dairy Journal, Edmonton, v. 14, n. 3, p. 263-268, 2004.

EIM, V. S.; SIMAL, S.; ROSSELLÓ, C.; FEMENIA, A. Effects of addition of carrot dietary fibre on the ripening process of a dry fermented sausage (sobrassada). Meat Science, Amsterdam, v. 80, n. 2, p. 173-182, 2008.

ELLEUCH, M.; BESBES, S.; ROISEUX, O.; BLECKER, C.; DEROANNE, C.; DRIRA, N.-E. Date flesh: Chemical composition and characteristics of the dietary fibre. Food Chemistry, London, v. 111, n. 3, p. 676-682, 2008.

ELLEUCH, M.; BEDIGIAN, D.; ROISEUX, O.; BESBES, S.; BLECKER, C.; ATTIA, H. Dietary fibre and fibre-rich by-products of food processing: Characterization, technological functionality and commercial applications: a review. Food Chemistry, London, v. 124, n. 2, p. 411-421, 2011.

FAO/WHO. Evaluation of health and nutritional properties of probiotics in food including powder milk with live lactic acid bacteria: report of a Joint FAO/ WHO Expert Consultation, Córdoba, 2001. Disponível em: $\quad<$ http::/www.ftp.fao.org/es/esn/food/probioreport en.pdf> . Acesso em: 21 jul. 2010.

FERNANDEZ-GARCIA, E.; MCGREGOR, J. U. Fortification of sweetened plain yogurt with insoluble dietary fiber. Zeitschrift fur Lebensmittel-Untersuchung und-Forschung A-Food Research and Technology, v. 204, n. 6, p. 433-437, 1997.

FERRAGUT, V.; CRUZ, N.S.; TRUJILLO, A.; GUAMIS, B.; CAPELLAS, M. Physical characteristics during storage of soy yogurt made from ultra-high pressure homogenized soymilk. Journal of Food Engineering, v. 92, n. 1, p. 63-69, 2009.

GARCÍA-PÉREZ, F. J.; SENDRA, E., LARIO, Y.; FERNÁNDEZ-LÓPEZ, J.; SAYAS, E.; PÉREZALVAREZ, J. A. Rheology of orange fiber enriched yogurt. Milchwissenschaft, Kempten, v. 61, n. 1, p. 5559, 2006.

GRANATO, D.; BRANCO, G. F.; NAZZARO, F.; CRUZ, A. G.; FARIA, J. A. F. functional foods and nondairy probiotic food development: trends, concepts and products. Comprehensive Reviews in food Science and Food Safety, v. 9, n. 3, p. 291-302, 2010.

GRIGELMO-MIGUEL, N.; CARRERASBOLADERAS, E.; MARTÍN-BELLOSO, O. Development of high-fruit-dietary-fibre muffins. European Food Research and Technology, v. 210, n. 2, p. 123-128, 1999.

GUIRGUIS, N.; BROOME, M. C.; HICKEY, M. W. The effect of partial replacement of skim milk powder with whey protein concentrate on the viscosity and syneresis of yoghurt. Australian Journal of Dairy Technology, v. 91, n. 1, p. 33-35, 1984.

GUZEL-SEYDIM, Z. B.; KOK-TAS, T.; GREENE, A. K.; SEYDIM, A. C. Review: functional properties of kefir. Critical Reviews in Food Science and Nutrition, v. 51, n. 3, p. 261-268, 2011.

IRIGOYEN, A.; ARANA, I., CASTIELLA, M.; TORRE, P.; IBÁNEZ, F.C. Microbiological, physicochemical, and sensory characteristics of kefir during storage. Food Chemistry, London. v. 90, n. 4, p. 613-620, 2005.

KOVALENKO, I. V.; BRIGGS, J. L. Textural characterization of soy-based yogurt by the vane method. Journal of Texture Studies, v. 33, n. 2, p. 105-118, 2002.

LARIO, Y.; SENDRA, E.; GARCIA-PEREZ, F. J.; FERNANDEZ- LOPEZ, J.; SAYAS-BARBERA, E.; PEREZ-ALVAREZ, J. A. Evolution of microbial populations of fiber enriched yogurt during commercial shelf-life. Milchwissenschaft-Milk Science International, v. 59, n. 11-12, p. 646-649, 2004. 
LOPITZ-OTSOA, F.; REMENTERIA, A.; ELGUEZABAL, N.; GARAIZAR, J. Kefir: asymbiotic yeasts-bacteria community with alleged healthy capabilities. Revista Iberoamericana de Micología, v. 23, p. 67-74, 2006.

LUCEY, J. A. The relationship between rheological parameters and whey separation in milk gels. Food Hydrocolloids, v. 15, n. 4-6, p. 603-608, 2001.

MAGENIS, R. B.; P RUDÊNCIO, E. S.; AMBONI, R. D. M. C.; CERQUEIRA JUNIOR, N. G.;OLIVEIRA, R. V. B.; SOLDI, V.; BENEDET, H. D. Compositional and physical properties of yogurt manufactured from milk and whey cheese concentred by ultrafiltration. International Journal of Food Science and Technology, London, v. 41, n. 5, p. 560-568, 2006.

McCANN, T. H.; FABRE, F.; DAY, L. Microstructure, rheology and storage stability of low-fat yoghurt structured by carrot cell wall particles. Food Research International, v. 44, n. 4, p. 884-892, 2011.

OKEZIE, B. O.; BELLO, A. B. Physicochemical and functional properties of Winged bean flour and isolate compared with soy isolate. Journal of Food Science, Chicago, v. 53, n. 2, p. 450-454, 1988.

ROBERTSON, J. A.; MONREDON, F. D.; DYSSELER, P.; GUILLON, F.; AMADO, R.; THIBAULT, J. F. Hydration properties of dietary fibre and resistant starch: a European collaborative study. LWT-Food Science and Technology, v. 33, n. 2, p. 72-79, 2000.

ROMANIN, D.; SERRADELL, M.; MACIEL, D. G.; LAUSADA, N.; GARROTE, G. L.; RUMBO, M. Downregulation of intestinal epithelial innate response by probiotic yeasts isolated from kefir. International Journal of Food Microbiology, v. 140, n. 2-3, p. 102-108, 2010.

SANGNARK, A.; NOOMHORM, A. Effect of particle sizes on functional properties of dietary fibre prepared from sugarcane bagasse. Food Chemistry, London, v. 80, n. 2, p. 221-229, 2003.
SENDRA, E.; KURI, V.; FERNANDEZ-LOPEZ, J.; SAYAS-BARBERA, E.; NAVARRO, C.; PEREZALVAREZ, J. A. Viscoelastic properties of orange fiber enriched yogurt as a function of fiber dose, size and thermal treatment. Food Science and Technology, v. 43, n. 4, p. 708-714, 2010.

SHAH, N. P. Functional cultures and health benefits. International Dairy Journal, Edmonton, v. 17, n. 1, p. 1262-77, 2007.

SIMOVA, E.; BESHKOVA, D.; ANGELOV, A.; HRISTOZOVA, T.; FRENGOVA, G.; SPASOV, Z. Lactic acid bacteria and yeasts in kefir grains and kefir made from them. Journal of Industrial Microbiology and Biotechnology, v. 28, n. 1, p. 1-6, 2002.

SIRÓ, I.; KAPOLNA, E.; KAPOLNA, B.; LUGASI, A. Functional food. Product development, marketing and consumer acceptance - a review. Appetite, v. 51, n. 3, p. 56-67, 2008.

STATSOFT Inc. STATISTICA (data analisys software system), version 7. Tulsa, Oklahoma, USA, 2004.

ÜNAL, B.; METIN, S.; I-IKLI, N. D. Use of response surface methodology to describe the combined effect of storage time, locust bean gum and dry matter of milk on the physical properties of low-fat set yoghurt. International Dairy Journal, v. 13, n. 11, p. 909-916, 2003.

VASILJEVIC, T.; KEALY, T.; MISHRA, V. K. Effects of beta-glucan addition to a probiotic containing yogurt. Journal of Food Science, Chicago, v. 72, n. 7, C405-C411, 2007.

VÁZQUEZ-OVANDO, A.; ROSADO-RUBIO, G.; CHEL-GUERRERO, L.; BETANCUR-ANCONA, D. Physicochemical properties of a fibrous fraction from chia (Salvia hispanica L.). LWT - Food Science and Technology, v. 42, n. 1, p. 168-173, 2009.

ZARE, F.; BOYE, J. I.; ORSAT, V.; CHAMPAGNE, C.; SIMPSON, B. K. Microbial, physical and sensory properties of yogurt supplemented with lentil flour. Food Research International, v. 44, n. 8, p. 2482-2488, 2011. 